\title{
Влияние интенсивности ионизирующего облучения на отклик МОП-структур
}

\author{
(C) O.В. Александров \\ Санкт-Петербургский государственный электротехнический университет „ЛЭТИ“, \\ 197376 Санкт-Петербург, Россия \\ E-mail: Aleksandr_ov@mail.ru \\ Поступила в Редакцию 8 октября 2020 г. \\ В окончательной редакции 10 октября 2020 г. \\ Принята к публикации 19 октября 2020 г.
}

\begin{abstract}
Проведено моделирование влияния интенсивности ионизирующего облучения на объемный заряд и плотность поверхностных состояний МОП-структур с тонким подзатворным диоксидом кремния. Показано, что зависимости плотности поверхностных состояний и объемного заряда от суммарного времени ионизирующего облучения и последующей выдержки при разных интенсивностях ионизирующего облучения ложатся на соответствующие общие кривые $N_{i t}(t)$ и $Q_{o t}(t)$. Общая кривая $N_{i t}(t)$ обусловлена дисперсионным характером транспорта ионов водорода $\mathrm{H}^{+}$. Наблюдаемые отклонения от общей кривой $N_{i t}(t)$ непосредственно после окончания ионизирующего облучения связаны с переходным процессом перераспределения ионов $\mathrm{H}^{+}$. Общая кривая $Q_{o t}(t)$ обусловлена релаксацией объемного заряда по механизму термоэмиссии с системы уровней с энергиями $0.3-1.0$ эВ. Показано, что повышенная чувствительность к малым дозам ионизирующего облучения (ELDRS) MOП-структур с толстым базовым оксидом при низких интенсивностях определяется дисперсионным характером транспорта ионов $\mathrm{H}^{+}$.
\end{abstract}

Ключевые слова: ионизирующее облучение, МОП-структура, поверхностные состояния, объемный заряд, дисперсионный транспорт, моделирование.

DOI: $10.21883 /$ FTP.2021.02.50502.9533

\section{1. Введение}

При ионизирующем облучении (ИО) МОП-структур образуются положительный объемный заряд (О3) в подзатворном диоксиде кремния и поверхностные состояния (ПС) на межфазной границе (МФГ) $\mathrm{Si}_{-} \mathrm{SiO}_{2}$ [1-3]. Образование объемного заряда связано с захватом дырок, образующихся при ИО, на дырочные ловушки кислородные вакансии в структуре $\mathrm{SiO}_{2}$. Образование ПС по модели МакЛина [4] объясняется освобождением под действием ИО ионов водорода $\mathrm{H}^{+}$в объеме $\mathrm{SiO}_{2}$, их транспортом к МФГ $\mathrm{Si}_{-} \mathrm{SiO}_{2}$ и депассивацией $P_{b} \mathrm{H}-$ центров по реакции [5]

$$
P_{b} \mathrm{H}+\mathrm{H}^{+} \rightarrow P_{b}+\mathrm{H}_{2} .
$$

После окончания ИО положительный О3 релаксирует вследствие эффектов туннелирования и термоэмиссии [6,7]. В отличие от О3, образование ПС продолжается длительное время после окончания ИО, что было объяснено в $[8,9]$ дисперсионным характером транспорта ионов $\mathrm{H}^{+}$в пленке аморфного диоксида кремния $\alpha-\mathrm{SiO}_{2}$. Установлено, что интенсивность ИО не оказывает влияния на зависимости объемного заряда $Q_{o t}(t)$ и плотности ПС $N_{i t}(t)$ в МОП-структурах с тонким подзатворным оксидом от суммарного времени ИО и последующей выдержки (отжига) [1-3,10,11]. В этом случае зависимости $N_{i t}(t)$ и $Q_{o t}(t)$ при разных интенсивностях и одной дозе ИО ложатся на соответствующие общие кривые [10,11]. Имеются, однако, отклонения $N_{i t}(t)$ от общей кривой непосредственно после окончания
ИО [11], не получившие объяснения. Такие отклонения приводят к зависимости среднего времени образования ПС от интенсивности ИО.

В биполярных приборах МОП-структуры имеют толстый базовый оксид при низкой напряженности электрического поля. У них наблюдается повышенная чувствительность к ИО при низких интенсивностях (Enhance Low Dose Rate Sensitivity (ELDRS)) (см. обзор [12]). Эффект ELDRS связывали с образованием объемного заряда [13-15] и с бимолекулярными реакциями рекомбинации свободных и связанных электронов и дырок [16,17]. С увеличением интенсивности ИО при наличии рекомбинационных центров возрастает скорость взаимной рекомбинации электронов и дырок и таким образом уменьшается суммарное количество захваченных дырок, отвечающих за образование О3 и ПС. Было, однако, показано [15], что механизм захвата дырок не позволяет объяснить все экспериментально наблюдаемые эффекты ELDRS. Авторами [15] была предложена количественная модель ELDRS, учитывающая разные подвижности дырок и ионов водорода. Согласно этой модели, поле, создаваемое дырками О3, является барьером для потока ионов водорода к МФГ $\mathrm{Si}-\mathrm{SiO}_{2}$, что приводит к уменьшению плотности ПС при высоких интенсивностях ИО. Однако модель [15] не позволяет объяснить наблюдаемого при этом значительного уменьшения величины О3.

В настоящей работе на основе численного решения системы диффузионно-кинетических уравнений, описывающих процесс образования ПС и О3, проведен 
анализ влияния интенсивности ИО на отклик МОПструктур с тонким подзатворным и толстым базовым оксидами.

\section{2. Описание модели}

В основе модели лежат известные реакции взаимодействия электронов и дырок, образующихся при ИО, с ловушками в подзатворном диоксиде кремния [18]. Дырочными ловушками являются нейтральные безводородные и водородосодержащие дефекты $T^{0}$ и $T \mathrm{H}^{0}$, где $T$ - дефект структуры диоксида кремния типа $\mathrm{O}_{3} \equiv \mathrm{Si}^{\bullet}$, обусловленный кислородной вакансией. При захвате дырки безводородной дырочной ловушкой образуется положительный объемный заряд:

$$
T^{0}+h^{+} \stackrel{k 1}{\longrightarrow} T^{+} .
$$

При последующем захвате электрона происходит нейтрализация положительно заряженной ловушки:

$$
T^{+}+e^{-\stackrel{k 2}{\longrightarrow}} T^{0}
$$

При взаимодействии дырки с водородосодержащей ловушкой образуется свободный ион водорода:

$$
T \mathrm{H}^{0}+h^{+} \stackrel{k 3}{\rightarrow} T^{0}+\mathrm{H}^{+} .
$$

Этот положительный ион водорода мигрирует к МФГ $\mathrm{Si}_{-} \mathrm{SiO}_{2}$, где освобождает от водорода (депассивирует) $P_{b}$-центр $\left(\mathrm{Si}_{3} \equiv \mathrm{Si}^{\bullet}\right)$, образуя таким образом ПС:

$$
\equiv \mathrm{SiH}+\mathrm{H}^{+} \rightarrow \equiv \mathrm{Si}^{\bullet+}+\mathrm{H}_{2} .
$$

Транспорт водорода в сетке аморфного диоксида кремния носит дисперсионный характер вследствие многократного захвата ионов $\mathrm{H}^{+}$на атомы мостикового кислорода и последующего освобождения с разными энергиями связи. В работах $[8,9]$ дисперсионный транспорт был описан моделью случайных блужданий (CTRW) c использованием эмпирических параметров. Нами было показано в [19], что дисперсионный транспорт ионов $\mathrm{H}^{+}$ в МОП-структурах может быть описан количественно на основе модели многократного захвата [20]. В соответствии с этой моделью непрерывное распределение локализованных состояний по энергиям заменяем на систему $k$ ловушек с дискретными уровнями $E_{b i}$, соответствующими энергии локализованных состояний или энергии связи иона $\mathrm{H}^{+}$с $i$-й ловушкой $S_{i}$. Захват ионов $\mathrm{H}^{+}$на ловушки происходит со скоростью $k_{f i}$, а освобождение со скоростью $k_{r i}$ согласно реакции:

$$
S_{i}+\mathrm{H}^{+} \underset{k r i}{\stackrel{k f i}{\rightleftarrows}} S_{i} \mathrm{H}^{+}, \quad i=1,2 \ldots k
$$

где $k$ - число локализованных состояний.

Поведение всех компонентов реакций $(1)-(5)$ в объеме подзатворного диэлектрика МОП-структуры при и после ИО с учетом дисперсионности транспорта ионов $\mathrm{H}^{+}$описываем системой диффузионно-кинетических уравнений непрерывности и уравнением Пуассона:

$$
\begin{gathered}
\frac{\partial p}{\partial t}=D_{p} \frac{\partial^{2} p}{\partial x^{2}}-\mu_{p} \frac{\partial}{\partial x}(p E)-\left(k_{1} C_{T}^{0}+k_{3} C_{T \mathrm{H}}^{0}\right) p+G \\
\frac{\partial n}{\partial t}=D_{n} \frac{\partial^{2} n}{\partial x^{2}}+\mu_{n} \frac{\partial}{\partial x}(n E)-k_{2} C_{T}^{+} n+G \\
\frac{\partial C_{\mathrm{H}}^{+}}{\partial t}=D_{\mathrm{H}}^{+} \frac{\partial^{2} C_{\mathrm{H}}^{+}}{\partial x^{2}}-\mu_{\mathrm{H}}^{+} \frac{\partial}{\partial x}\left(C_{\mathrm{H}}^{+} E\right)+k_{3} C_{T \mathrm{H}}^{0} p \\
-C_{\mathrm{H}}^{+} \sum_{i=1}^{k} k_{f i} C_{S i}^{0}+\sum_{i=1}^{k} k_{r i} C_{S \mathrm{H} i}^{+} \\
\frac{\partial C_{S \mathrm{H} i}^{+}}{\partial t}=-\frac{\partial C_{S i}^{0}}{\partial t}=k_{f i} C_{S i}^{0} C_{\mathrm{H}}^{+}-k_{r i} C_{S \mathrm{H} i}^{+}, i=1,2 \ldots \\
\frac{\partial C_{T}^{+}}{\partial t}=k_{1} C_{T}^{0} p-k_{2} C_{T}^{+} n-R \\
\frac{\partial C_{T}^{0}}{\partial t}=\left(k_{3} C_{T \mathrm{H}}^{0}-k_{1} C_{T}^{0}\right) p+k_{2} C_{T}^{+} n+R \\
\frac{\partial C_{T \mathrm{H}}^{0}}{\partial t}=-k_{3} C_{T \mathrm{H}}^{0} p \\
\frac{\partial^{2} V}{\partial x^{2}}=-\frac{\varepsilon_{0}}{\varepsilon_{0}}\left(C_{T}^{+}+C_{\mathrm{H}}^{+}+p-n+\sum_{i=1} C_{S \mathrm{H} i}^{+}\right)
\end{gathered}
$$

где $t$ - время, отсчитываемое от начала ИО; $x$ - координата, отсчитываемая от кремниевой подложки $(x=0)$ до затвора $(x=d, d-$ толщина подзатворного диэлектрика); $n$ и $p$ - концентрации свободных электронов и дырок соответственно; $D_{n}$ и $\mu_{n}$ - коэффициенты диффузии и подвижность электронов $\left(\mu_{n}=20 \mathrm{~cm}^{2} / \mathrm{B} \cdot \mathrm{c}[21]\right)$; $D_{p}$ и $\mu_{p}$ - коэффициент диффузии и подвижность дырок $\left(D_{p}=2 \cdot 10^{-7} \mathrm{~cm}^{2} /\right.$ В.с [22] $) ; C_{T}^{0}, C_{T}^{+}$и $C_{T \mathrm{H}}^{0}-$ концентрации нейтральных и положительно заряженных безводородных и нейтральных водородосодержащих ловушек; $C_{\mathrm{H}}^{+}$- концентрации свободных ионов $\mathrm{H}^{+} ; D_{\mathrm{H}}^{+}$и $\mu_{\mathrm{H}}^{+}-$ их коэффициент диффузии и подвижность; $C_{S i}^{0}$ и $C_{S H i}^{+}-$ концентрации $i$-х ловушек пустых и с локализованными на них ионами $\mathrm{H}^{+}$соответственно; $V$ - распределение потенциала в диоксиде кремния, $E$ - напряженность электрического поля, $E=-d V / d x ; q-$ заряд электрона; $\varepsilon$ - относительная диэлектрическая проницаемость диоксида кремния $(\varepsilon=3.9) ; \varepsilon_{0}$ - диэлектрическая постоянная. Темп генерации электронно-дырочных пар $G$ определяется мощностью радиационной дозы $F$, коэффициентом генерации электронно-дырочных пар $k_{g}$ и вероятностью $f_{y}(E)$ разделения пар электрическим полем до их начальной рекомбинации: $G(E)=F k_{g} f_{y}(E)$. Темп генерации рассчитывался по [23] при коэффициенте генерации электронно-дырочных пар, соответствующем типу ИО. 
Рекомбинационный член $R$ в уравнении (10) обусловлен разрядкой положительно заряженных ловушек $T^{+}$ посредством туннелирования [6,7]:

$$
R_{T N}=\alpha \exp (-\beta(E) x)
$$

где $\alpha$ - частотный фактор, $\beta(E)$ - барьерный фактор, или посредством термоэмиссии $[6,7]$,

$$
R_{T E}=A T^{2} \exp \left(-E_{T E} / k_{\mathrm{B}} T\right),
$$

где $E_{T E}$ - высота потенциального барьера, $A-$ постоянная Ричардсона $\left(A=120 \mathrm{~A} / \mathrm{cm}^{2} \mathrm{~K}^{2}[7]\right), k_{\mathrm{B}}$ - постоянная Больцмана, $T$ - температура $(T=300 \mathrm{~K})$.

Полагаем, что в начальный момент времени концентрации всех компонентов нулевые:

$$
n(x, 0)=p(x, 0)=0 ; \quad C_{T}^{+}(x, 0)=C_{\mathrm{H}}^{+}(x, 0)=0 .
$$

Исходные концентрации нейтральных безводородных дырочных ловушек распределены вблизи подложки по экспоненциальному закону:

$$
C_{T 0}^{0}(x)=Q_{T 0}^{0} / l_{T} \exp \left(-x / l_{T}\right)
$$

а водородосодержащие ловушки имеют однородную составляющую,

$$
C_{T \mathrm{H} 0}^{0}(x)=Q_{T \mathrm{H} 0}^{0} / l_{T \mathrm{H}}\left[1+B \exp \left(-x / l_{T \mathrm{H}}\right)\right],
$$

где $Q_{T 0}^{0}$ и $Q_{T H 0}^{0}$ - исходные интегральные концентрации безводородных и водородосодержащих дырочных ловушек соответственно, $l_{T}$ и $l_{T \mathrm{H}}-$ ширина их распределений (полагали $\left.l_{T}=l_{T \mathrm{H}}=5 \mathrm{Hм}\right), B-$ долевой коэффициент приграничной составляющей концентрации водородосодержащих ловушек.

Уравнения (6), (7) решались при граничных условиях, соответствующих нулевой концентрации свободных носителей на обеих межфазных границах:

$$
p(0, t)=p(d, t)=n(0, t)=n(d, t)=0 .
$$

Для ионов $\mathrm{H}^{+}$граница с подложкой при $x=0$ полагается поглощающей, а граница с затвором при $x=d-$ отражающей:

$$
C_{\mathrm{H}}^{+}(x, t)=0 ; \quad j_{\mathrm{H}}^{+}(d, t)=0,
$$

где $j_{\mathrm{H}}^{+}-$поток ионизованного водорода,

$$
j_{\mathrm{H}}^{+}=-D_{\mathrm{H}}^{+} \frac{\partial C_{\mathrm{H}}^{+}}{\partial x}+\mu_{\mathrm{H}}^{+} C_{\mathrm{H}}^{+} E .
$$

К затвору относительно подложки приложено напряжение $V_{g}$ :

$$
V(0, t)=0 ; \quad V(d, t)=V_{g} .
$$

Ловушки для ионов $\mathrm{H}^{+}$связаны с нарушениями структуры $\alpha-\mathrm{SiO}_{2}$, полагаем располагаются равномерно по толщине диэлектрика:

$$
C_{S i}^{0}(x, 0)=C_{S i}^{0}\left(E_{b i}\right) .
$$

В начальный момент все водородные ловушки пустые:

$$
C_{S \mathrm{H} i}^{0}(x, 0)=0 .
$$

Полагаем, что ионы $\mathrm{H}^{+}$захватываются на ловушки с одинаковой скоростью: $k_{f i}=k_{f}=\sigma_{\mathrm{H}}^{+} v_{d}$, где $\sigma_{\mathrm{H}}^{+}-$ сечение захвата ионов $\mathrm{H}^{+}$на ловушки, $v_{d}-$ дрейфовая скорость, $v_{d}=\mu_{\mathrm{H}}^{+} E$. Освобождение ионов $\mathrm{H}^{+}$с ловушек происходит со скоростью, зависящей от энергии связи иона с ловушкой $E_{b i}, k_{r i}=v \exp \left(-\frac{E_{b i}}{k_{\mathrm{B}} T}\right)$, где $v-$ частотный фактор, соответствующий частоте колебаний атомов $\left(v==10^{12} \mathrm{c}^{-1}\right)$. Распределение плотности локализованных состояний по энергии имеет экспоненциальный вид, характерный для некристаллических и аморфных материалов [24]:

$$
C_{S i}^{0}\left(E_{b i}\right)=\frac{C_{S}^{0}}{E_{0}} \exp \left(-\frac{E_{b i}}{E_{0}}\right)
$$

где $C_{S}^{0}-$ полная концентрация водородных ловушек, $C_{S}^{0}=\sum_{i=1}^{k} C_{S i}^{0} ; E_{0}-$ характеристическая энергия, соответствующая ширине энергетического распределения ловушек, связанная с дисперсионным параметром $\alpha$ соотношением $\alpha=k_{\mathrm{B}} T / E_{0}$.

Сдвиг порогового напряжения под действием облучения $\left(\Delta V_{t h}\right)$ складывается из объемной $\left(\Delta V_{o t}\right)$ и поверхностной $\left(\Delta V_{i t}\right)$ составляющих:

$$
\Delta V_{t h}=\Delta V_{o t}+\Delta V_{i t}=-\left(Q_{o t}+Q_{i t}\right) / C_{o x},
$$

где $C_{o x}$ - удельная емкость диэлектрика, $C_{o x}=\varepsilon \varepsilon_{0} / d$, $Q_{i t}$ - заряд ПС, $Q_{o t}$ - эффективный объемный заряд,

$$
\left.Q_{o t}=\frac{1}{d} \int_{0}^{d} C_{T}^{+}(x) d-x\right) d x .
$$

Заряд ПС определяется плотностью ПС, которая определяется суммарным количеством ионизованного водорода, стекающим на МФГ $\mathrm{Si}-\mathrm{SiO}_{2}$ :

$$
N_{i t}=\int_{0}^{t} j_{\mathrm{H}}^{+}(0, t) d t .
$$

В модели использовались следующие значения параметров: $D_{\mathrm{H}}^{+}=1.0 \exp \left(-\frac{0.73}{k_{\mathrm{B}} T}\right) \quad[25] ; \quad E_{0}=0.07$ эВ $\left(\alpha=k_{\mathrm{B}} T / E_{0}=0.37\right)$, что соответствует литературному диапазону $\left(\alpha=0.32-0.38\right.$ для $\mathrm{H}^{+} \quad$ в $\left.\mathrm{SiO}_{2}[8,9]\right)$. Полагаем, что сечение захвата ионов $\mathrm{H}^{+}$на локализованные состояния $\sigma_{\mathrm{H}}^{+}$зависит от напряженности электрического поля таким же образом, как сечение захвата дырок $\sigma_{p}$ в $\mathrm{SiO}_{2}$ в сильных полях (при $E \gtrsim 1 \mathrm{MB} / \mathrm{cm}): \sigma_{p} \sim E^{-a}$, где $a=0.4-0.6[26,27]$. Принимаем поэтому $\sigma_{\mathrm{H}}^{+}=\gamma E^{-0.5}$, где коэффициент $\gamma$ был определен из сопоставления с экспериментальными зависимостями: $\gamma=7.5 \cdot 10^{-10} \mathrm{~B}^{0.5} \mathrm{~cm}^{1.5}$, что соответствует $\sigma_{\mathrm{H}}^{+}=8 \cdot 10^{-13} \mathrm{~cm}^{2}$ при $E=1 \mathrm{MB} / \mathrm{cм}$. Минимальная $E_{b 1}=0.80$ эВ (при $E=1 \mathrm{MB} / \mathrm{cm}$ ) и максимальная $E_{b k}=0.98$ эВ энергии локализованных состояний 
определялись из сопоставления с экспериментальными зависимостями при равномерном распределении энергий внутри диапазона: $E_{b i}=E_{b 1}+\left(E_{b k}-E_{b 1}\right) i / k$. Число локализованных состояний принималось $k \geq 10$, когда решение переставало зависеть от $k$. Константы скоростей реакций (1)-(3): $k_{1,3}=\sigma_{p 1,3} V_{t h} D_{p} / D_{n} ; k_{2}=\sigma_{n 2} V_{t h}$, где $\sigma_{p}$ и $\sigma_{n}$ - сечения захвата дырок и электронов соответственно, $v_{t h}$ - тепловая скорость электронов, $v_{t h} \cong 10^{7} \mathrm{~cm} / \mathrm{c}$. Учитывалась зависимость от напряженности электрического поля сечений захвата дырок и электронов по работам [27,28] при значениях сечения захвата в слабых полях дырок $\sigma_{p 01}=2.1 \cdot 10^{-13} \mathrm{~cm}^{2}$, $\sigma_{p 03}=1.4 \cdot 10^{-14} \mathrm{~cm}^{2}$ и электронов $\sigma_{n 02}=8 \cdot 10^{-14} \mathrm{~cm}^{2}$, как в работе [18]. Таким образом, варьируемыми параметрами модели являются исходные интегральные концентрации водородосодержащих $Q_{T \mathrm{H} 0}^{0}$ и безводородных $Q_{T 0}^{0}$ дырочных ловушек, а также полная концентрация водородных ловушек $C_{S}^{0}$, зависящие от конкретной технологии выращивания подзатворного диэлектрика.

\section{3. Результаты расчетов и их обсуждение}

Уравнения модели (6)-(13) решались численно с использованием неявной и явной разностных схем с начальными условиями $(16)-(18),(23)$ и граничными условиями (19)-(23) с учетом (24)-(27). Решения сравнивались с экспериментальными зависимостями плотности поверхностных состояний и объемного заряда в МОП-транзисторах от суммарного времени ИО и последующей выдержки при различных интенсивностях ИО, полученными в работах $[10,11]$.

После окончания импульса ИО $(G=0)$ электроны и дырки, имеющие высокие подвижности, покидают подзатворный диэлектрик, а освобожденные ионы $\mathrm{H}^{+}$ под действием электрического поля $\left(+V_{G}\right.$ на затворе $)$ дисперсионно переносятся к кремниевой подложке, образуя ПС по реакции (3). Зависимости плотности ПС при нескольких интенсивностях ИО от суммарного времени ИО и последующей выдержки при положительной полярности $V_{G}$ как при, так и после ИО $(E=1 \mathrm{MB} / \mathrm{cm})$ показаны на рис. 1. На рисунке приведены экспериментальные данные работы [11], полученные на линейном ускорителе электронов (LINAC) до энергии 10 Мэв с разным числом импульсов длительностью 10 мкс и частотой повторения 7.5 Гц (значки $1-3$ ) с одинаковой дозой 75 крад $\left(\mathrm{SiO}_{2}\right)$. Расчетные кривые $\left(1^{\prime}-3^{\prime}\right)$ получены с учетом дисперсии уровней ловушек для ионов $\mathrm{H}^{+}$от $E_{b 1}=0.77$ эВ до $E_{b k}=0.99$ эВ. Величина $E_{b 1}$ определяет начало, а $E_{b k}$ - конец роста плотности ПС во времени. Как видно из рисунка, плотность ПС монотонно растет как во время ИО (пунктирные линии), так и после окончания ИО (сплошные линии). Через некоторое время после окончания ИО зависимости при разных интенсивностях ИО ложатся на одну общую кривую,

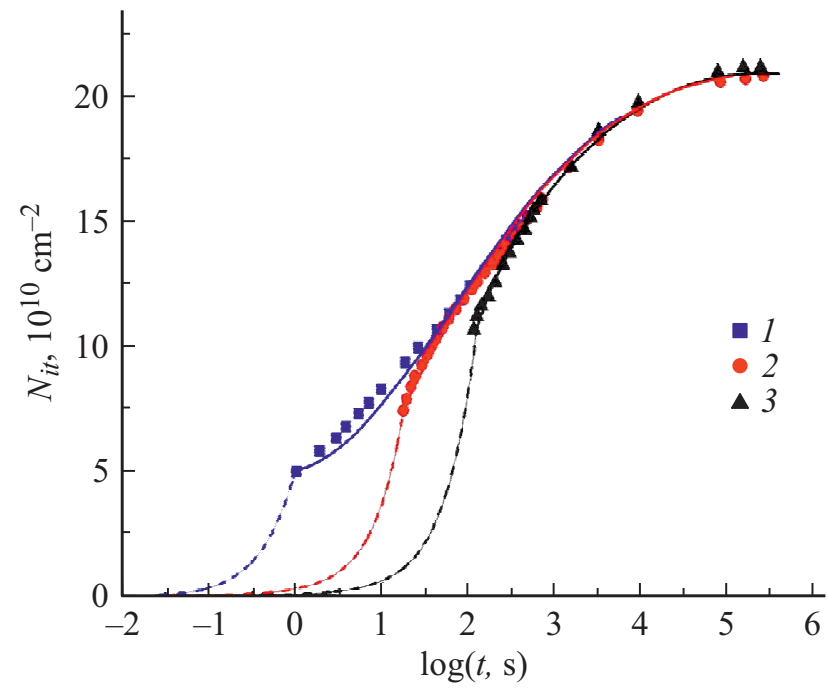

Рис. 1. Кинетика накопления плотности ПС при эффективной мощности дозы ИО: $1-3-$ эксперимент (LINAC) [11], рад/с: $1-5$ имп. по $1.4 \cdot 10^{9} ; 2-70$ имп. по $1.1 \cdot 10^{8} ; 3-572$ имп. по $1.3 \cdot 10^{7} ; 1^{\prime}-3^{\prime}-$ расчет при ИО (пунктирные линии) и после ИО (сплошные линии), рад/с: $1^{\prime}-6 \cdot 10^{4} ; 2^{\prime}-4.3 \cdot 10^{3}$; 3 - 520. $\left(Q_{\mathrm{TH}}^{0}=6.7 \cdot 10^{12} \mathrm{~cm}^{-2}, B=3, C_{S}^{0}=8 \cdot 10^{22} \mathrm{~cm}^{-3}\right.$, $D=75$ крад, $\left.d=47.1 \mathrm{Hм}, V_{G}=+4.71 \mathrm{~B}\right)$.

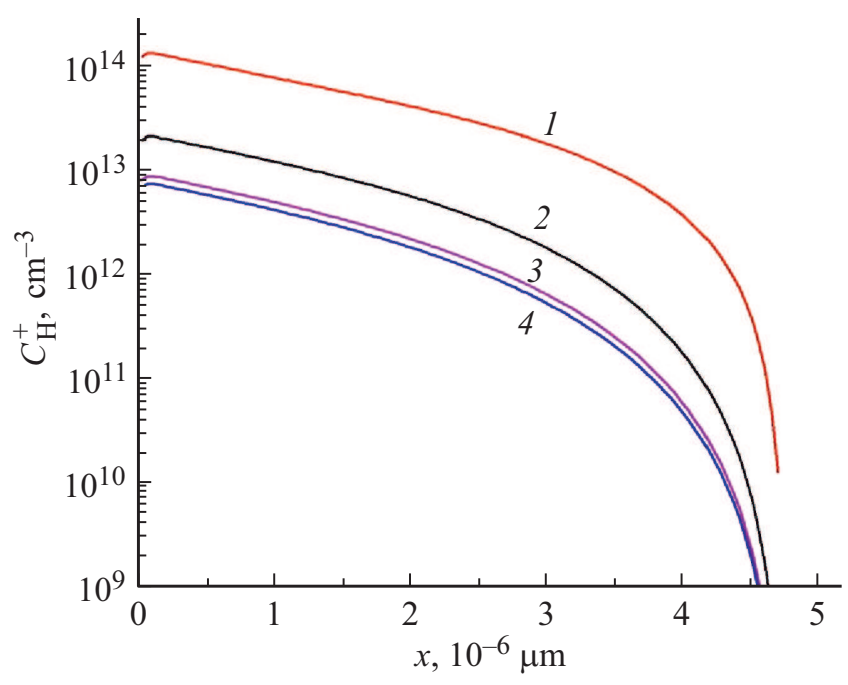

Рис. 2. Распределения концентрации ионов $\mathrm{H}^{+}$по толщине $\mathrm{SiO}_{2}$ после ИО и выдержки в течение суммарного времени, с: $1,2-125 ; 3,4-300$ при эффективной мощности дозы ИО, рад/с: $1, .3-520 ; 2,4-4.3 \cdot 10^{3}$. Параметры расчета как на рис. 1.

тогда влияние интенсивности ИО от суммарного времени исчезает. Однако непосредственно после окончания ИО наблюдается отклонение временны́х зависимостей плотности ПС от общей кривой.

Распределения концентраций свободных ионов $\mathrm{H}^{+}$ по толщине подзатворного диэлектрика при разных интенсивностях ИО, но при одинаковых суммарных временах ИО и последующей выдержки показаны на 
рис. 2. Как видно из рисунка, концентрация свободных ионов $\mathrm{H}^{+}$с интенсивностью 600 рад/с непосредственно после окончания ИО при 125 с (кривая 1) выше, чем с интенсивностью $4.3 \cdot 10^{3}$ рад/с после ИО и выдержки с тем же суммарным временем (кривая 2). При увеличении суммарного времени до $300 \mathrm{c}$, когда плотности ПС при разных интенсивностях ИО ложатся на одну общую кривую, распределения ионов $\mathrm{H}^{+}$по толщине примерно совпадают. Таким образом, при одинаковом количестве освобожденного при ИО водорода, при одинаковой дозе ИО, непосредственно после окончания ИО концентрация свободного водорода в диэлектрике выше (а плотность ПС соответственно ниже), чем концентрация, соответствующая общей кривой. Этот результат объясняется переходным процессом перераспределения ионов $\mathrm{H}^{+}$от состояния генерации электронно-дырочных пар по всему объему диэлектрика $(G \neq 0)$ к состоянию отсутствия такой генерации $(G=0)$. Перераспределение осуществляется посредством транспорта ионов $\mathrm{H}^{+}$ в электрическом поле диэлектрика, который замедлен вследствие дисперсионного характера переноса.

Влияние ИО на изменение поверхностной $V_{i t}$ и объемной $V_{o t}$ составляющих порогового напряжения во время и после ИО при различных интенсивностях ИО показаны на рис. $3, a, b$ соответственно. На рисунке приведены экспериментальные данные работы [10], полученные при облучении на линейном ускорителе электронов до энергии 20 МэВ с числом импульсов 2 , длительностью 8 мкс и частотой повторения 4Гц (значки 1 ), а также при рентгеновском облучении ( $X$-ray 10 кэВ, значки 2,3 ) с одинаковой дозой 100 крад $\left(\mathrm{SiO}_{2}\right)$. Как видно из рис. $3, a$, расчетные зависимости роста $V_{i t}$ при различных интенсивностях (кривые $1^{\prime}-3^{\prime}$ ) так же, как и на рис. 1, через некоторое время после окончания ИО ложатся на общую кривую.

Модуль объемной составляющей $\left|V_{o t}\right|$ на рис. $3, b$ (кривые $1^{\prime}-3^{\prime}$ ) растет с увеличением интенсивности ИО вследствие роста концентрации дырок. При дальнейшей выдержке после ИО $\left|V_{o t}\right|$ уменьшается вследствие релаксации ОЗ. В отличие от ПС изменение O3 начинается через короткое время после окончания ИО (при $t \sim 3 \cdot 10^{-1}$ с на рис. $3, b$ ). По данным работы [28], разрядка О3 начинается уже при $t \sim 10^{-3}$ с. Принято считать, что до температуры $\sim 125^{\circ} \mathrm{C}$ релаксация О3 после ИО происходит преимущественно по механизму туннелирования, а при более высоких температурах по механизму термоэмиссии [6,29]. Однако наши расчеты показывают, что при механизме туннелирования $\left(R=R_{T N}\right.$ по (14)), с параметрами, близкими к найденным в [30] $\left(\alpha=1.5 \cdot 10^{-2} \mathrm{c}^{-1}, \beta(E)=\right.$ $=\beta_{0}\left(1-\gamma E^{0.5}\right), \quad \beta_{0}=2.0 \cdot 10^{7} \mathrm{~cm}^{-1}, \quad \gamma=8 \cdot 10^{-7} \mathrm{~cm} / \mathrm{B}$, что при $x_{0} \cong l_{T}=5$ нм соответствует высоте барьера $E_{T} \cong 1.5$ эВ), релаксация начинается только при достаточно больших временах после окончания ИО - при $t>100$ с (кривая $0^{\prime \prime}$ на рис. $\left.3, b\right)$. Это происходит, когда фронт туннелирования, идущий от подложки, захватит заметную часть О3, распределенного по толщине
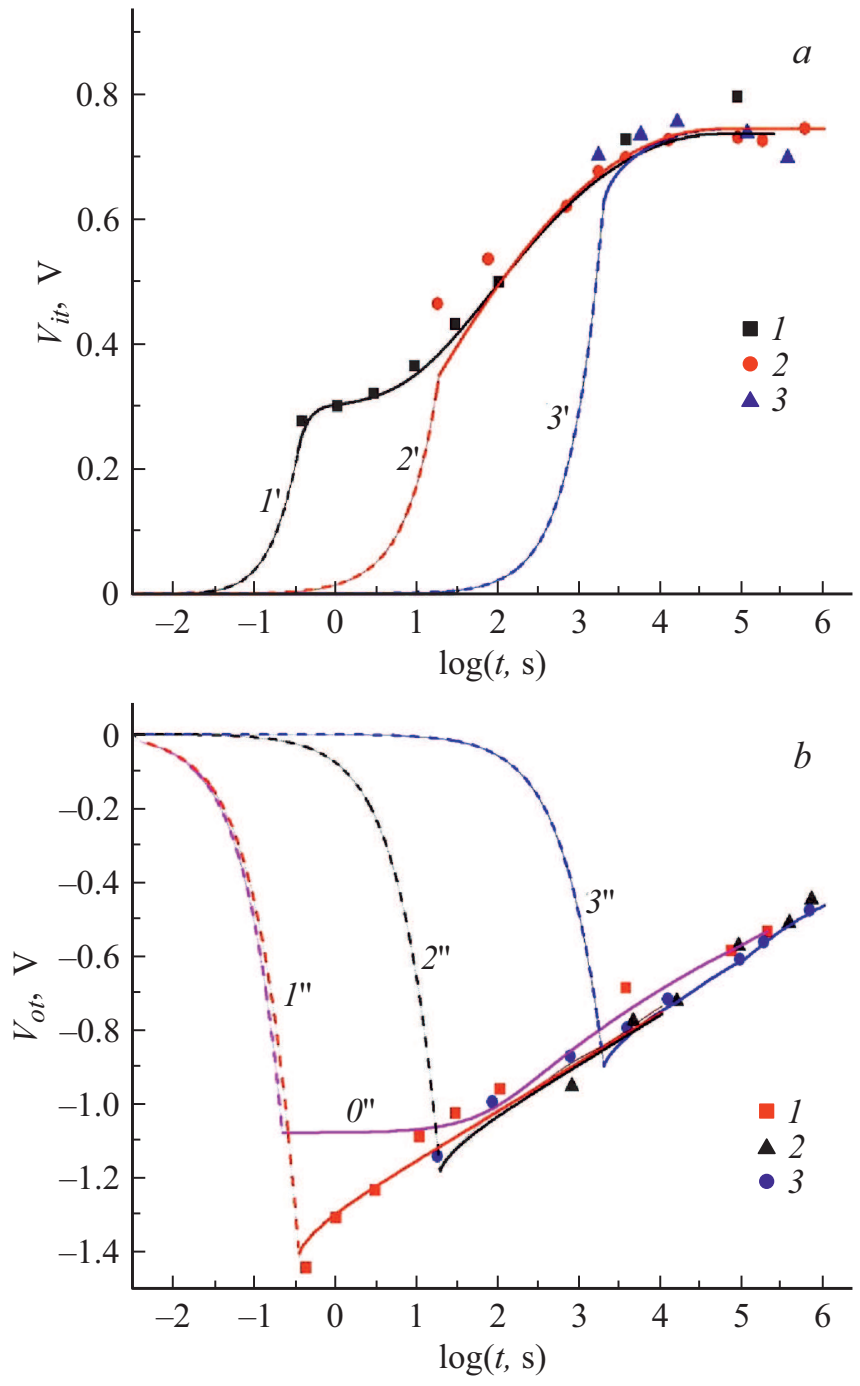

Рис. 3. $a, b-$ кинетика изменения поверхностной $V_{i t}(a)$ и объемной $V_{o t}(b)$ составляющих порогового напряжения при интенсивности ИО, рад/с: $1,1^{\prime}, 0^{\prime \prime}, 1^{\prime \prime}-5 \cdot 10^{5} 2$ имп. LINAC по $\left.6 \cdot 10^{9}\right) ; 2,2^{\prime}, 2^{\prime \prime}-5.5 \cdot 10^{3}(X$-ray $) ; 3,3^{\prime}, 3^{\prime \prime}-$ $5.2 \cdot 10^{1}$ (X-ray); $1-3-$ эксперимент $[10] ; 1^{\prime}-3^{\prime}-$ расчет $V_{i t} ; O^{\prime \prime}-3^{\prime \prime}$ - расчет $V_{o t}$ при ИО (пунктирные линии) и после ИО (сплошные линии), $0^{\prime \prime}-R=R_{T N}, 1^{\prime \prime}-3^{\prime \prime}-$ $R=R_{T E} .\left(Q_{T \mathrm{H}}^{0}=2.6 \cdot 10^{12} \mathrm{~cm}^{-3}, B=3.3, Q_{T}^{0}=6.3 \cdot 10^{12} \mathrm{~cm}^{-3}\right.$, $C_{S}^{0}=2 \cdot 10^{22} \mathrm{~cm}^{-3} \cdot D=100$ крад, $\left.d=60 \mathrm{HM}, V_{G}=+6 \mathrm{~B}\right)$.

пленки. Следовательно, механизмом релаксации О3 является не туннелирование, а термоэмиссия $\left(R=R_{T E}\right.$ по (15)). Для описания экспериментальной кривой оказалось необходимым введение семейства энергетических уровней термоэмиссии в диапазоне от $E_{T E 1}=0.3$ эВ до $E_{T E k}=1.0$ эВ (кривые $\left.1^{\prime \prime}-3^{\prime \prime}\right)$. В этом случае уравнение (10) приобретает вид

$$
\frac{\partial C_{T J}^{+}}{\partial t}=k_{1} C_{T j}^{0} p-k_{3} C_{T j}^{+} n-R_{T E j}, \quad j=1, \ldots k,
$$

где индекс $j$ в (28) соответствует $j$-й составляющей компонента с энергией термоэмиссии 


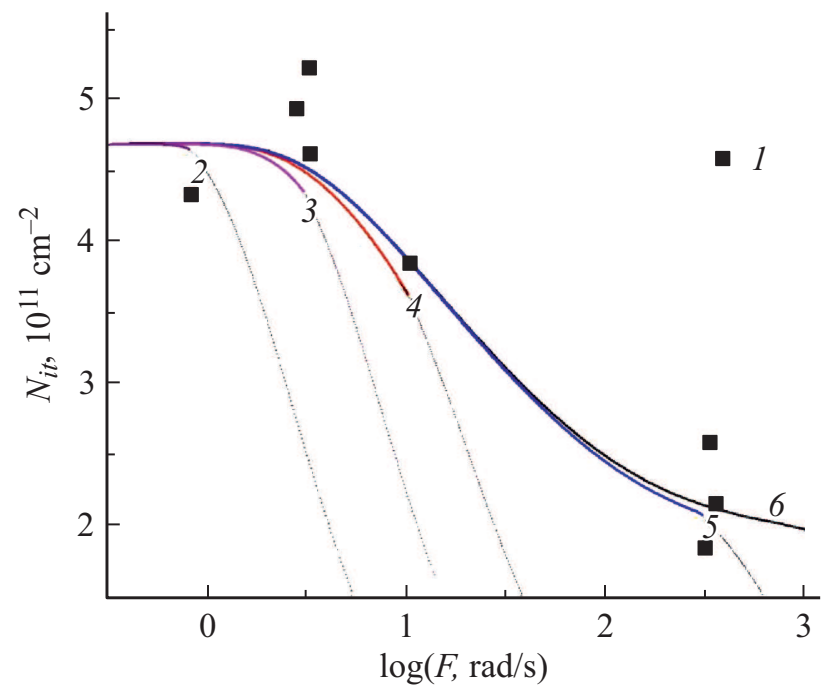

Рис. 4. Зависимость плотности ПС от интенсивности ИО. 1 - эксперимент [15], 2-6 - расчет при мощности дозы, рад/с: $2-0.8,3-3,4-10,5-300,6-$ общая кривая. $\left(Q_{T \mathrm{H}}^{0}=1.5 \cdot 10^{11} \mathrm{~cm}^{-2}, B=3.3, C_{S}^{0}=2.3 \cdot 10^{21} \mathrm{~cm}^{-3}\right.$, $D=200$ крад, $d=600$ нм, $E=0.01 \mathrm{MB} / \mathrm{cm})$.

$E_{T E j}=E_{T E 1}+\left(E_{T E k}-E_{T E 1}\right) j / k$ и начальной концентрацией $C_{T 0 j}^{0}(x)=C_{T 0}^{0}(x) j / k$. В уравнениях $(6),(7),(11)$ и (13) $C_{T}^{+}$заменятся на $\sum_{j=1}^{k} C_{T j}^{+}$, а $C_{T}^{0}$ на $\sum_{j=1}^{k} C_{T j}^{0}$.

При малых временах разряжаются положительно заряженные ловушки с малыми энергиями, а при больших - с большими, при этом разрядка О3 происходит сразу по всей толщине пленки. Отметим, что по литературным данным [6] уровни положительно заряженных дырочных ловушек лежат в диапазоне от $E_{C}+0.8$ эВ до $E_{C}+1.35$ эВ при нулевой напряженности электрического поля, что с учетом понижения барьера в электрическом поле $\left(\Delta E_{T} \cong E l_{T}=0.5\right.$ эВ при $l_{T}=5 \mathrm{Hм}$ и $E=1 \mathrm{MB} / \mathrm{cm}$ ) примерно соответствует найденному диапазону энергий термоэмиссии.

Особенностью МОП-структур в биполярных приборах являются большая толщина базового оксида и низкая напряженность электрического поля. В этом случае образующийся при захвате дырок на ловушки О3 может менять распределение и направление электрического поля, что полагается причиной ELDRS в моделях $[13,14]$. Отметим, что даже при малых или нулевых напряжениях на затворе вследствие наличия собственного фиксированного заряда $Q \sim 10^{11} \mathrm{~cm}^{-2}$ в термическом диоксиде кремния имеется внутреннее электрическое поле $E=q Q / \varepsilon \varepsilon_{0} \sim 0.01 \mathrm{MB} /$ см. Зависимости плотности ПС от интенсивности ИО для МОП-структуры с толщиной оксида 600 нм при таком поле $(E=0.01 \mathrm{MB} / \mathrm{cm})$ показаны на рис. 4. На рисунке приведены экспериментальные данные из работы [15], полученные при рентгеновском (10 кэВ) ИО с мощностью дозы от 0.8 до 300 рад/с (значки 1). Расчетные кривые 2-6 получены при экспоненциальном распределении $C_{T \mathrm{H} 0}^{0}(x) \mathrm{c} l_{T}=80 \mathrm{Hм}$, значи- тельно большем, и при $C_{S}^{0}=2.3 \cdot 10^{21} \mathrm{~cm}^{-3}$, на порядок меньшем, чем для тонкого подзатворного оксида, что является, по-видимому, спецификой толстых оксидов. Минимальная энергия $E_{b 1}=0.9$ эВ при $E=0.01 \mathrm{MB} / \mathrm{cm}$ соответствует зависимости $E_{b 1}$ от напряженности электрического поля [19]. Как видно из рисунка, расчетные кривые удовлетворительно описывают экспериментальные данные. Таким образом, зависимость плотности ПС от интенсивности ИО для МОП-структур с толстым базовым оксидом, так же как и с тонким подзатворным, определяется дисперсионным транспортом ионов $\mathrm{H}^{+}$.

\section{4. Заключение}

Проведено численное моделирование влияния интенсивности ионизирующего облучения (ИО) на величину объемного заряда и плотность поверхностных состояний МОП-структур во время и после окончания ИО. Показано, что при разных интенсивностях ИО зависимости плотности ПС и О3 от суммарного времени ИО и выдержки ложатся на соответствующие общие кривые $N_{i t}(t)$ и $Q_{o t}(t)$. Общая кривая $N_{i t}(t)$ обусловлена дисперсионным характером транспорта ионов водорода. Отклонение зависимости $N_{i t}(t)$ от общей кривой обусловлено переходным процессом перераспределения ионов $\mathrm{H}^{+}$ от состояния генерации электронно-дырочных пар к состоянию отсутствия генерации. Кинетика релаксации объемного заряда при временах $\lesssim 100$ с не может быть описана механизмом туннелирования. Во всем диапазоне времен она удовлетворительно описывается механизмом термоэмиссии системы уровней с энергиями в диапазоне 0.3-1.0 эВ. Показано, что зависимость плотности ПС от интенсивности ИО для МОП-структур с толстым базовым оксидом, так же как и с тонким подзатворным, определяется дисперсионным транспортом ионов $\mathrm{H}^{+}$.

\section{Конфликт интересов}

Автор заявляет, что у него нет конфликта интересов.

\section{Список литературы}

[1] D.M. Fleetwood. IEEE Trans. Nucl. Sci., 65 (8), 1465 (2018).

[2] К.И. Таперо, В.Н. Улимов, А.М. Членов. Радиационные эфбекты в кремниевых интегральных схемах космического применения (М., БИНОМ, 2012).

[3] T.R. Oldham, F.B. McLean. IEEE Trans. Nucl. Sci., 50 (3), 483 (2003).

[4] F.B. McLean. IEEE Trans. Nucl. Sci., 27 (6), 1651 (1980).

[5] E. Cartier, J.H. Stathis, D.A. Buchanan. Appl. Phys. Lett., 63 (11), 1510 (1993).

[6] P.J. McWhorter, S.L. Miller, W.M. Miller. IEEE Trans. Nucl. Sci., 37 (6), 1682 (1990).

[7] В.А. Гуртов, П.А. Райкерус, А.А. Сарен. Зарядоперенос в структурах с диэлектрическими слоями (Петрозаводск, Изд-во ПетрГУ, 2010). 
[8] N.S. Saks, D.B. Brown. IEEE Trans. Nucl. Sci., 36 (6), 1848 (1989).

[9] D.B. Brown, N.S. Saks. J. Appl. Phys., 70 (7), 3734 (1991).

[10] D.M. Fleetwood, P.S. Winokur, J.R. Schwank. IEEE Trans. Nucl. Sci., 35 (6), 1497 (1988).

[11] M.R. Shaneyfelt, J.R. Schwank, D.M. Fleetwood, P.S. Winokur, K.L.Hughes, G.L. Hash, M.P. Connors. IEEE Trans. Nucl. Sci., 39 (6), 2244 (1992).

[12] R.L. Pease, R.D. Schrimpf, D.M. Fleetwood. IEEE Trans. Nucl. Sci., 56 (4), 1894 (2009).

[13] S.C. Witczak, R.C. Lacoe, D.C. Mayer, D.M. Fleetwood, R.D. Schrimpf, K.F. Galloway. IEEE Trans. Nucl. Sci., 45 (6), 2339 (1998).

[14] R.J. Graves, C.R. Cirba, R.D. Schrimpf, R.J. Milanowski, A. Michez, D.M. Fleetwood, S.C. Witczak, F. Saigne. IEEE Trans. Nucl. Sci., 45 (6), 2352 (1998).

[15] S.N. Rashkeev, C.R. Cirba, D.M. Fleetwood, R.D. Schrimpf, S.C. Witczak, A. Michez, S.T. Pantelides. IEEE Trans. Nucl. Sci., 49 (6), 2650 (2002).

[16] H.P. Hjalmarson, R.L. Pease, R.A.B. Devine. IEEE Trans. Nucl. Sci., 55 (6), 3009 (2008).

[17] J. Boch, F. Saigne, A.D. Touboul, S. Ducret, J.-F. Carlotti, M. Bernard, R.D. Schrimpf, F. Wrobel, G. Sarrabayrouse. Appl. Phys. Lett., 88, 232113 (2006).

[18] О.В. Александров. ФТП, 49 (6), 793 (2015).

[19] О.В. Александров. ФТП, 55 (10), 1029 (2020).

[20] J. Noolandi. Phys. Rev. B, 16 (10), 4466, 4474 (1977).

[21] R.C. Huges. Phys. Rev. Lett., 30, 1333 (1973).

[22] R.C. Hughes. Phys. Rev. B, 15 (4), 2012 (1977).

[23] J.M. Benedetto, H.E. Boesch. IEEE Trans. Nucl. Sci., 33 (6), 1318 (1986).

[24] V.I. Arkhipov, A.I. Rudenko. Phill. Mag. B, 45 (2), 189, 209 (1982).

[25] S.R. Hofstein. IEEE Trans. Electron Dev., 14 (11), 749 (1967).

[26] J.J. Tzou, J.Y.-C. Sun, C.-T. Sah. Appl. Phys. Lett., 43 (9), 861 (1983).

[27] R.J. Krantz, L.W. Aukerman, T.C. Zietlow. IEEE Trans. Nucl. Sci., 34 (6), 1196 (1987).

[28] H.E. Boesch, F.B. McLean, J.M. Benedetto, J.M. McGarrity. IEEE Trans. Nucl. Sci., 33 (6), 1191 (1986).

[29] A.J. Lelis, T.R. Oldham, H.E. Boesch, F.B. McLean. IEEE Trans. Nucl. Sci., 36 (6), 1808 (1989).

[30] О.В. Александров. ФТП, 54 (2), 181 (2020).

Редактор Г.А. Оганесян

\section{linfluence of ionizing radiation intensity on the response of MOS-structures}

\section{O.V. Aleksandrov}

\section{St.Petersburg State Electrotechnical University „LETl“, 197376 St. Petersburg, Russia}

Abstract The influence of the intensity of ionizing radiation on the volume charge and the density of surface states of MOSstructures with thin gate silicon dioxide was modeled. It is shown that the dependencies of density of surface states and volume charge on total time of the ionizing radiation and subsequent anneal at different ionizing radiation IR intensities lie on the corresponding general curves $N_{i t}(t)$ and $Q_{o t}(t)$. The general curve $N_{i t}(t)$ is is determined due to the dispersive nature of the transport of hydrogen ions $\mathrm{H}^{+}$. The observed deviations from this general curve immediately after the end of ionizing radiation are related to the transition process of $\mathrm{H}^{+}$ion redistribution. The general curve $Q_{o t}(t)$ is due to the relaxation of the volume charge by the mechanism of thermo-emission from a system of levels with energies of $0.3-1.0 \mathrm{eV}$. It is shown that enhance low dose rate sensitivity (ELDRS) for MOS structures with a thick base oxide is determined by the dispersion transport of $\mathrm{H}^{+}$ions. 